\title{
Map of dimorphic switching-related signaling pathways in Sporothrix schenckii based on its transcriptome
}

\author{
FANGLIANG ZHENG ${ }^{1}$, WEI GAO ${ }^{1}$, YING WANG ${ }^{1}$, QINGYAN CHEN $^{1}$, \\ QIULING ZHANG ${ }^{2}$, XIUYAN JIANG ${ }^{1}$, BINBIN HOU $^{3}$ and ZHENYING ZHANG ${ }^{4}$ \\ ${ }^{1}$ Academy of Life Science, Liaoning University, Shenyang, Liaoning $110036 ;{ }^{2}$ Department of Dermatology, \\ Shenzhen Shekou People's Hospital, Shenzhen, Guangdong 518067; ${ }^{3}$ Department of Dermatology, \\ The Second Hospital of Dalian Medical University, Dalian, Liaoning 116021; ${ }^{4}$ Department of Dermatology, \\ University of Hong Kong Shenzhen Hospital, Shenzhen, Guangdong 518000, P.R. China
}

Received August 27, 2020; Accepted May 24, 2021

DOI: $10.3892 / \mathrm{mmr} .2021 .12285$

\begin{abstract}
Sporothrix schenckii (S. schenckii) induces sporotrichosis, which has gained attention in recent years due to its worldwide prevalence. The dimorphic switching process is essential for the pathogenesis of $S$. schenckii. Previously, overexpression of several signal transduction genes, including SsDRK1 and SsSte20, was observed during the mycelium-to-yeast transition; these were necessary for asexual development, yeast-phase cell formation, cell wall integrity and melanin synthesis. However, the mechanisms of the signaling pathways during dimorphic switching of S. schenckii remain unclear. In the present study, transcriptome sequencing of the 48-h induced yeast forms and mycelium of S. schenckii was performed. In total, 24,904,510 high-quality clean reads were obtained from mycelium samples and 22,814,406 from 48-h induced yeast form samples. Following assembly, 31,779 unigene sequences were obtained with $52.98 \%$ GC content (The proportion of guanine $\mathrm{G}$ and cytosine $\mathrm{C}$ to all bases in nucleic acid). The results demonstrated that 12,217 genes, including genes involved in signal transduction and chitin synthesis, were expressed differentially between the two stages. According to these results, a map of the signaling pathways, including two-component and heterotrimeric G-protein signaling systems, Ras and MAPK cascades associated with the dimorphic switch, was drawn. Taken together, the transcriptome data and analysis performed in the present study
\end{abstract}

Correspondence to: $\operatorname{Dr}$ Zhenying Zhang, Department of Dermatology, University of Hong Kong Shenzhen Hospital, 1 Haiyuan Road, Shenzhen, Guangdong 518000, P.R. China

E-mail: zhangzy@hku-szh.org

Dr Binbin Hou, Department of Dermatology, The Second Hospital of Dalian Medical University, 467 Zhongshan Road, Dalian, Liaoning 116021, P.R. China

E-mail: houbinbin1001@163.com

Key words: signaling pathways, Sporothrix schenckii, dimorphic switching, pathogenesis, transcriptome lay the foundation for further research into the molecular mechanisms controlling the dimorphic switch of S. schenckii and support the development of anti-S. schenckii strategies targeting genes associated with signaling pathways.

\section{Introduction}

Sporotrichosis, an implantation mycosis caused mainly by the dimorphic fungus Sporothrix schenckii (S. schenckii), has gained attention over the two last decades due to its broad geographic range and prevalence in tropical and subtropical areas $(1,2)$. Sporotrichosis, which is caused by transcutaneous trauma, may progress into chronic cutaneous, subcutaneous or even deeper infections that may involve lymphatic tissue, fascia, muscles, cartilage and bones $(1,3)$. After S. schenckii implants in a host via skin wounds, the marked changes in its environment, including in the temperature, $\mathrm{pH}$, osmotic pressure and nutrients, exert pressure, causing it to adapt to the new environment by transforming from the mycelium phase to the yeast phase and settling down (4). Dimorphic switching, which requires that the fungus sense and respond to stimuli in the host environment, is necessary for establishing its pathogenicity (5). A number of signaling pathways, including the two-component and heterotrimeric G-protein signaling systems, as well as Ras and cAMP signaling and the downstream mitogen-activated protein kinase (MAPK) cascades have been found to induce or influence the dimorphic switch (6-11). Dozens of genes in these signaling pathways in Histoplasma capsulatum, Talaromyces marneffei, Blastomyces dermatitidis and Paracoccidioides brasiliensis, four dimorphic pathogenic fungi with higher morbidity and mortality rates than $S$. schenckii have been investigated to identify the key determinants of pathogenicity and dimorphic switching using mutagenesis or RNAi knockdown techniques $(6,10,12,13)$. In our previous study, it was demonstrated that SsDRK1 in the two-component system and SsSte20 in the Ras signaling pathway were overexpressed during the early yeast stage, but not in the mycelial stage, of $S$. schenckii using two-dimensional electrophoresis (14). Furthermore, it was demonstrated that SsDRK1 is essential for normal asexual development, yeast-phase cell formation, cell wall composition and integrity, and melanin synthesis using 
double-stranded RNA interference mediated by Agrobacterium tumefaciens (14-17). However, the details of the signaling pathways controlling dimorphic switching remain unclear due to the limited literature available regarding S. schenckii.

In the present study, the transcriptomics of the 48 -h induced yeast and mycelial stages of $S$. schenckii underwent transcriptome analysis. Signaling pathways associated with the dimorphic switch, including the two-component and heterotrimeric G-protein signaling systems, Ras, and MAPK cascades were mapped using the Kyoto Encyclopedia of Genes and Genomes (KEGG) based on comparative transcriptomic results between $48-\mathrm{h}$ induced yeast and mycelial cells. In addition, the cell wall ultrastructural features of 48-h induced yeast cells were compared with those of mycelial cells. The results provided novel insights into the molecular mechanisms controlling the dimorphic switch in S. schenckii.

\section{Materials and methods}

Fungal strain and culture conditions. The strain of S. schenckii used, ATCC10268, was maintained at the Research Center for Pathogenic Fungi, Liaoning University, China. To obtain a mycelial culture, the ATCC10268 isolate was inoculated onto Sabouraud dextrose agar (SDA) solid medium (10 g/l tryptone, $40 \mathrm{~g} / 1$ glucose) and incubated at $25^{\circ} \mathrm{C}$. The mycelial colonies subsequently obtained were inoculated in liquid Sabouraud medium and cultured with shaking at $100 \mathrm{rpm}$ at $25^{\circ} \mathrm{C}$ for $48 \mathrm{~h}$. To induce the switch of $S$. schenckii from the mycelial phase to the 48-h induced yeast phase, mycelial culture was enriched and transferred to brain-heart infusion (BHI) liquid medium (HyClone; GE Healthcare Life Sciences) (18-23), which was incubated at $37^{\circ} \mathrm{C}$ and shaken at $100 \mathrm{rpm}$ for $48 \mathrm{~h}$.

Transmission electron microscopy (TEM). S. schenckii cultures grown in SDA at $25^{\circ} \mathrm{C}$ or in $\mathrm{BHI}$ at $37^{\circ} \mathrm{C}$ for $48 \mathrm{~h}$ were collected, and the cell suspensions were fixed at $4^{\circ} \mathrm{C}$ by addition of an equal volume of fixing solution ( $0.2 \mathrm{M} \mathrm{Na}_{2} \mathrm{HPO}_{4}, 0.2 \mathrm{M}$ $\mathrm{NaH}_{2} \mathrm{PO}_{4}$ ) for 2-4 h. Cells were transferred to a centrifuge tube and spun at $12,000 \mathrm{x}$ g for $15 \mathrm{~min}$ at $4^{\circ} \mathrm{C}$ to obtain a cell pellet, which was embedded in $1 \%$ agarose and then washed in $0.1 \mathrm{M}$ PBS three times for $15 \mathrm{~min}$ each. Post-fixation staining was carried out with $1 \% \mathrm{OsO}_{4}$ in $0.1 \mathrm{M} \mathrm{PBS}(\mathrm{pH} 7.4$ ) for $2 \mathrm{~h}$ at room temperature, followed by removal of $\mathrm{OsO}_{4}$, rinsing in 0.1 M PBS (pH 7.4) three times, and dehydration. Following infiltration and embedding the cell pellet, ultrathin sections (60-80 nm) were cut with an ultramicrotome. Sections were stained with uranyl acetate in pure ethanol for $15 \mathrm{~min}$ at $4^{\circ} \mathrm{C}$ rinsed with distilled water, stained with lead citrate for $15 \mathrm{~min}$ at $4^{\circ} \mathrm{C}$, and rinsed with distilled water. The sections were allowed to air-dry overnight and were then observed using TEM (24). The thicknesses of the inner and outer layers of the mycelial cell wall were measured using Image-Pro Plus 6.0 (Media Cybernetics, Inc.).

Fluorescence staining of fungi. S. schenckii cultured in SDA at $25^{\circ} \mathrm{C}$ or in $\mathrm{BHI}$ at $37^{\circ} \mathrm{C}$ for $48 \mathrm{~h}$ were smeared onto slides and stained with fungal fluorescence dyes (Lifetime bio) that bind specifically to chitin and cellulose for $15 \mathrm{~min}$ at $4^{\circ} \mathrm{C}$. The slides were visualized using a Nikon 2000 fluorescent microscope (Nikon Instruments, Inc.) with a x20 objective. For each slide, 10 microscopic fields were examined and the fields with median fluorescent brightness selected for comparation. Images were processed with NIS-Elements (version 4.10; Nikon Instruments, Inc.) imaging software.

cDNA library construction and sequencing. S. schenckii in mycelial and 48-h induced yeast forms were collected for RNA extraction. Total RNA was extracted using RNAiso ${ }^{\mathrm{TM}}$ plus (Takara Bio, Inc.) and treated with RNase-free DNase I (Fermentas; Thermo Fisher Scientific, Inc.) to remove residual DNA. The quantity of RNA was determined using NanoDrop ND-1000 (Thermo Fisher Scientific, Inc.) and 1.2\% agarose gels. The integrity of the total RNA was assessed using an Agilent 2200 Tape Station (Agilent Technologies, Inc.), and each sample had an RNA integrity number $>7.5$.

cDNA libraries were constructed using the NEBNext ${ }^{\circledR}$ Ultra $^{\mathrm{TM}}$ RNA Library Prep Kit for Illumina (Illumina, Inc.) according to the manufacturer's protocols. After the total RNA was extracted, the mRNA was purified using poly-T oligo-attached magnetic beads and fragmented into small pieces in fragmentation buffer from the kit. Using mRNA as a template, first-strand cDNA was synthesized using random primers and reverse transcriptase. Second-strand cDNA was synthesized using RNase $\mathrm{H}$ and DNA polymerase I (Takara Bio, Inc.) (25). Subsequently, the cDNA was purified, and subjected to end-repair, poly(A) addition, sequencing, adapter connecting and fragment size selection. Finally, the purified cDNA was PCR amplified and sequenced on the Illumina HiSeq $^{\mathrm{TM}}$ Xten platform (Guangzhou RiboBio Co., Ltd.).

Transcriptome de novo assembly and annotation. After analyzing the base composition and quality value, the sequencing data were filtered according to the raw data analysis results to remove the adaptor sequences, contaminated parts and low-quality reads to obtain clean reads. Next, clean reads were de novo assembled using Trinity (version no. v2013-02-25; Trinity Software, Inc.). Trinity software consists of three independent software modules, Inchworm, Chrysalis and Butterfly, which are used in turn to process large-scale RNA-Seq read data and obtain unigene sequences.

After assembly, the unigene sequences were annotated into the NCBI non-redundant (NR; http://www.ncbi.nlm.nih. gov/), Swiss-Prot (http://www.expasy.ch/spot/), Clusters of eukaryotic Orthologous Group (KOG; http://www.ncbi.nlm. nih.gov/cog/) and Kyoto Encyclopedia of Genes and Genomes (KEGG; http://www.geno me.jp/kegg) databases using the BLASTALL package (release 2.2.28) from NCBI with an E-value $\leq 10^{-5}$. Based on NR annotation, GO functional annotation and further functional classification were performed using Blast2GO (http://www.blast2go.com/b2ghome).

Identification of differentially expressed genes (DEGS) and functional analysis. Bowtie2 (version no. 2.2.5) was used to map the clean reads back to the unigenes, and the unigene mapping rate was counted. The unigene expressed values and transcript levels were calculated by the fragments per kilobase of transcript per million mapped reads (FPKM) method using the mapping results (26). EdgeR (version no. 3.10.0) was used to calculate the expression difference, and multiple hypothesis testing was performed to correct the P-value of the difference 
A

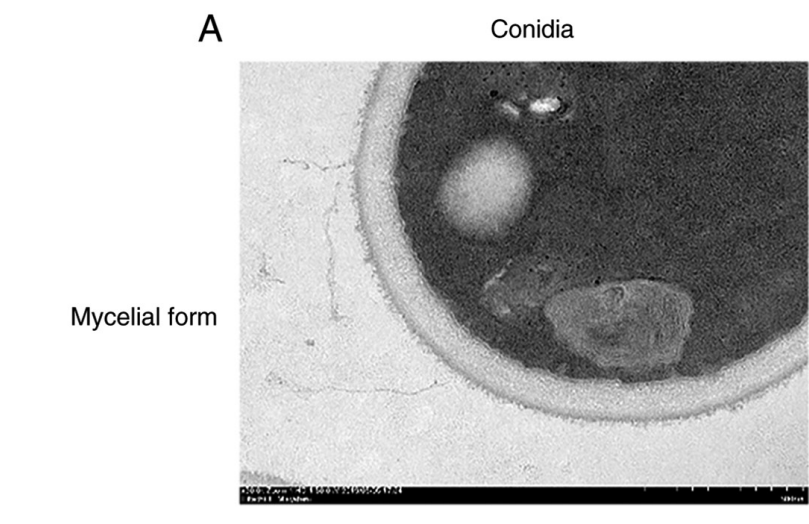

C

48-h induced yeast

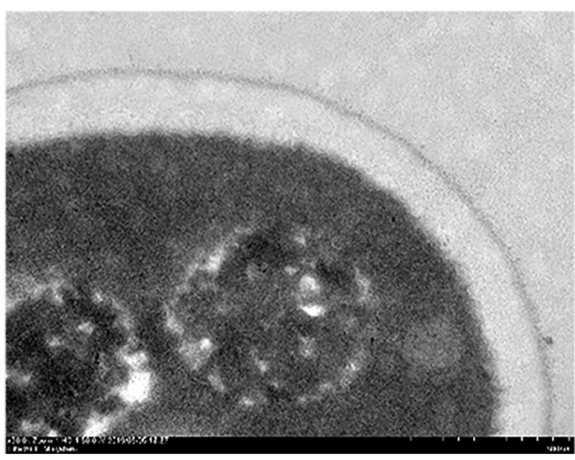

B

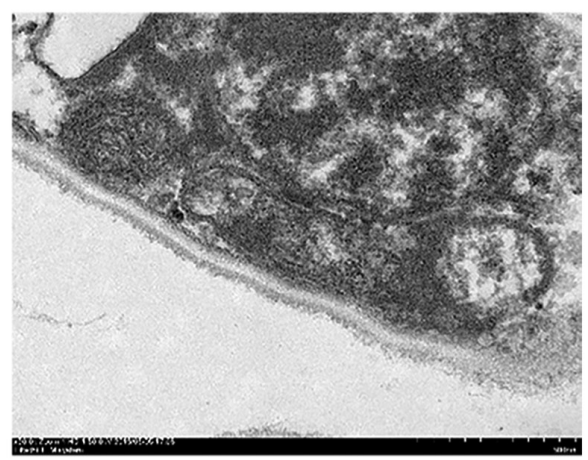

D

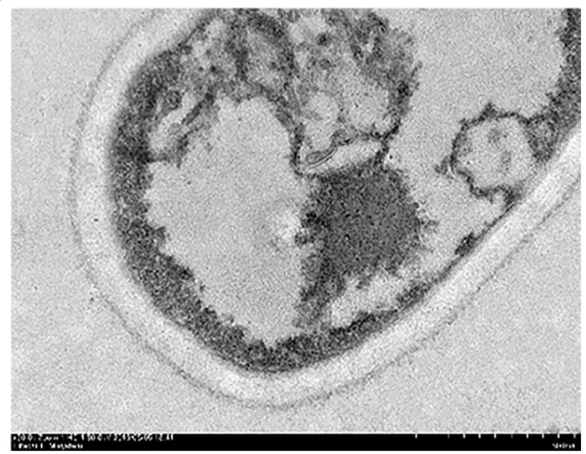

Figure 1. TEM of images of mycelial and yeast cells of S. schenckii showing the double-layer cell wall decorated with thin fibrils. (A) Conidium of the mycelial form of $S$. schenckii; the inner layer of the cell wall was $102 \mathrm{~nm}$, and the outer layer was $36 \mathrm{~nm}$ thick. (B) Mycelium of the mycelial form of $S$. schenckii; the inner layer of the cell wall was $44 \mathrm{~nm}$ thick, and the outer layer was $40 \mathrm{~nm}$ thick. (C) Conidium of the 48-h induced yeast form of $S$. schenckii; the inner layer of the cell wall was $151 \mathrm{~nm}$ thick, and the outer layer was $32 \mathrm{~nm}$ thick. (D) Mycelium of the 48 -h induced yeast form of S. schenckii; the inner layer of the cell wall was $101 \mathrm{~nm}$ thick, and the outer layer was $25 \mathrm{~nm}$ thick. TEM, transmission electron microscopy; S. schenckii, Sporothrix schenckii.

A

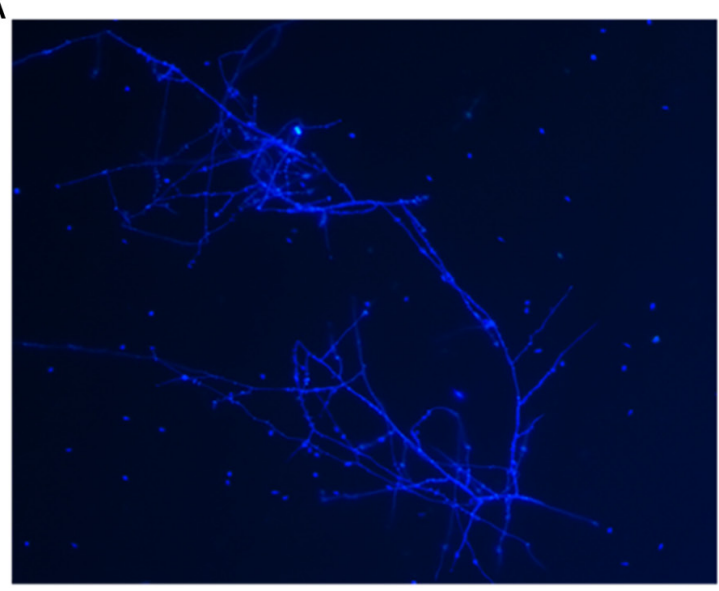

Mycelia cells
B

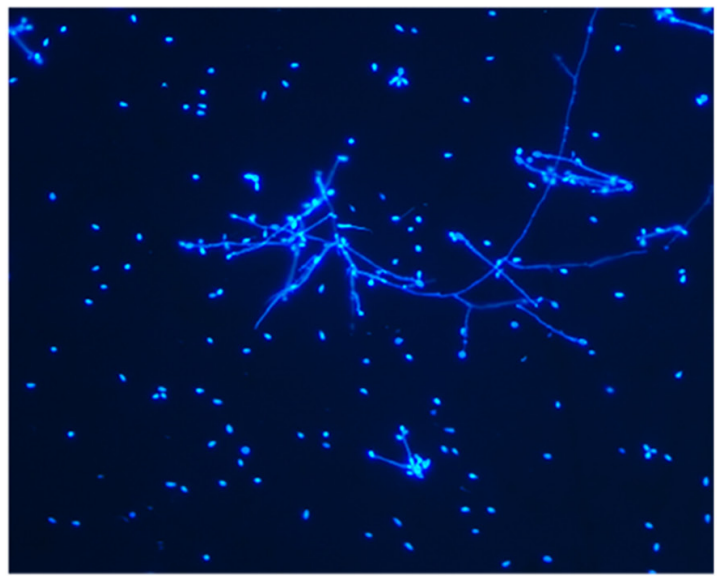

48-h induced yeast

Figure 2. Fluorescence microscopy images of S. schenckii (A) mycelial cells and (B) 48-h induced yeast cells labeled with fungal fluorescence dye. The labeled cells were visualized using a Nikon Microscope (x20 magnification). S. schenckii, Sporothrix schenckii.

test. The threshold P-value was determined by controlling the false discovery rate (FDR). When the FDR value of the difference test was obtained, the differential expression multiples of the gene in different samples were calculated according to the amount of gene expression, using $\log _{2} \mathrm{FCl} \geq 1$, and FDR $<0.05$ as the threshold for screening differential genes (27).

The hypergeometric distribution test was used for GO classification and KEGG analysis of the DEGs to understand the functional properties of the genes and regulatory pathways involved. The obtained GO categories with $\mathrm{P}<0.05$ and pathways with q-value $\leq 0.05$ were defined as significantly enriched GO classifications and KEGG pathways (28).

Statistical analysis. One-way analysis of variance was performed using SPSS 17.0 (SPSS, Inc.). Differences were tested with Duncan's test, unless otherwise specified. 
Table I. Sequencing data statistics.

\begin{tabular}{lccccrr}
\hline Sample & Raw reads & Q20 $(\%)$ & Q30 $(\%)$ & Err(\%) & GC\% & Clean reads \\
\hline Mycelial & $27,465,858$ & 95.70 & 90.91 & 0.33 & 54.84 \\
48-h induced yeast & $23,746,248$ & 97.66 & 94.69 & 0.18 & 46.80 & $22,804,510$ \\
\hline
\end{tabular}

Q20, an error rate of 1 in 100, with a corresponding call accuracy of 99\%; Q30, represent an error rate of 1 in 1,000, with a corresponding call accuracy of $99.9 \%$; Err, error rate.

Table II. Assembly result statistics.

\begin{tabular}{|c|c|c|c|c|c|c|}
\hline Total_sequence & Total_base & N50 & N90 & Max_length & Min_length & $\mathrm{GC}(\%)$ \\
\hline 31,779 & $72,164,889$ & 4,155 & 993 & 29,705 & 301 & 52.98 \\
\hline
\end{tabular}

$\mathrm{N} 50$, a standard to evaluate the quality of genome splicing. The unigenes are sorted from large to small, when the length of fragments is accumulated to $50 \%$ of the total fragment length, the length of small fragments is the N50 value; N90, a standard to evaluate the quality of genome splicing. The unigenes are sorted from large to small, when the length of fragments is accumulated to $90 \%$ of the total fragment length, the length of small fragments is the N50 value.

\section{Results}

Cell wall structures. The structural organization of hyphal and conidial cell walls of S. schenckii in the mycelial and 48-h induced yeast forms was studied using high-pressure freezing TEM (HPF-TEM). Fewer hyphal fragments and larger conidia were observed when comparing the character of 48-h induced yeast cells with that of mycelial cells scanned with HPF-TEM. The cell wall of S. schenckii was observed as having a double-layered structure, with a low electron-density inner layer and a high electron-density outer layer, decorated with thin fibrils. The inner layer thicknesses of the S. schenckii mycelial cell wall was $\sim 102 \mathrm{~nm}$, thinner than the $151 \mathrm{~nm}$ in 48-h induced yeast cells, which decreased the total thickness of the mycelial cell wall. Meanwhile, compared with the 48-h induced yeast form, the mycelial cell walls showed a thicker outer layer densely covered with fine fibrils (Fig. 1).

To confirm the difference in cell wall structure between the mycelial and 48-h induced yeast forms, the cells were stained with Calcofluor White, targeting the chitin in the cell wall of S. schenckii. The fluorescence pattern observed in 48-h induced yeast cells, compared with that in mycelial cells, had stronger chitin and cellulose labeling patterns on the 48-h induced yeast cell surface (Fig. 2).

Transcriptome sequencing data processing and assembly result statistics. Following sequencing two samples of the mycelial and 48-h induced yeast phases using the Illumina platform, the mycelial phase samples were sequenced to obtain $27,465,858$ reads and $4.12 \mathrm{G}$ data were obtained, in which the Q20 and Q30 base proportions were 95.70 and 90.91\%, respectively. The GC content proportion was $54.84 \%$ and the $\mathrm{N}$ ratio in sequencing was $0.33 \%$. Sequencing the 48 -h induced yeast samples gave 23,746,248 reads and 3.56 G of data, with Q20 and Q30 base ratios of 97.66 and $94.69 \%$, respectively, a GC
Table III. Transcriptome data functional annotation statistics.

Annotation Database Number of unigenes Percentage (\%)

\begin{tabular}{lcc}
\hline Nr & 21,079 & 66.33 \\
Swissprot & 17,398 & 54.75 \\
KOG & 13,854 & 43.59 \\
KEGG & 11,062 & 34.81 \\
In at least one database & 23,329 & 73.41 \\
Total unigenes & 31,779 & 100.0 \\
\hline
\end{tabular}

$\mathrm{Nr}$, Non-redundant protein database; KOG, the eukaryotic database of Clusters of Orthologous Group of proteins database; KEGG, Kyoto Encyclopedia of Genes and Genomes.

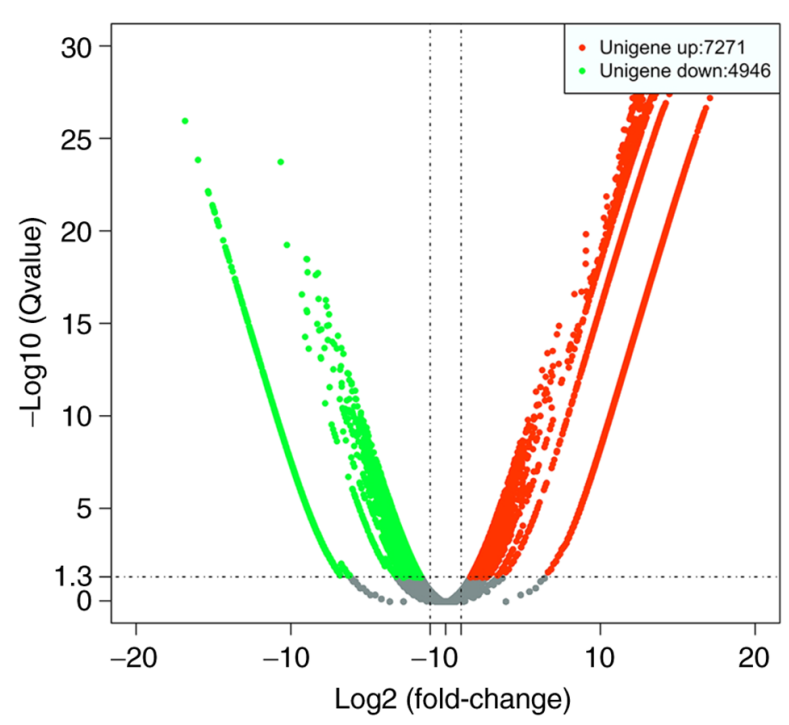

Figure 3. Volcano plot of differentially-expressed genes. In total, 12,217 genes were differentially expressed between the mycelial and 48-h induced yeast forms, with 7,271 genes upregulated and 4,946 genes downregulated. 


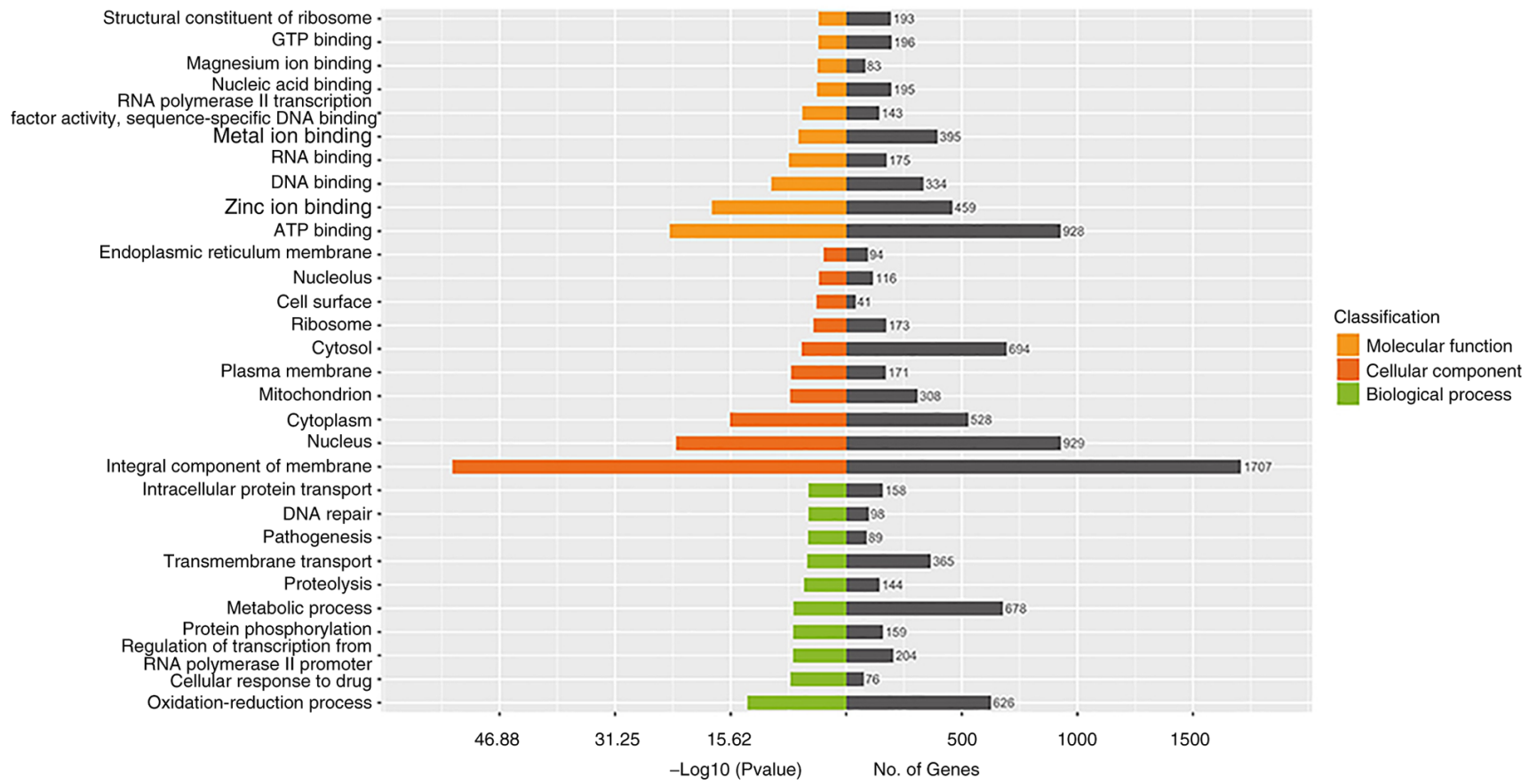

Figure 4. GO enrichment analysis of common differentially-expressed genes. The horizontal coordinate represents enriched GOs, and the vertical coordinate indicates the number of differentially-expressed genes. Different colors represented different GO classifications, namely molecular function, biological process and cellular component. In this map, enriched genes in the cellular component classification were most abundant and were mainly concentrated in the 'integral component of membrane' class. GO, Gene Ontology.

content ratio of $46.80 \%$, and $\mathrm{N}$ ratio in sequencing of $0.18 \%$. Subsequently, quality filtration of the raw data was performed. Mycelial phase samples gave 24,904,510 clean reads, and 48-h induced yeast phase samples gave 22,814,406 clean reads (Table I).

Trinity was used to assemble high-quality sequences in the samples. Following assembly, 31,779 unigene sequences were obtained. The total number of sequences generated was $72.2 \mathrm{Mbp}$, and the N50 and N90 lengths were 4,155 and $993 \mathrm{bp}$, respectively. The Max-length and Min-length of the unigenes were 29,705 and $301 \mathrm{bp}$, respectively, and the GC content was $52.98 \%$ (Table II). The transcriptomic data supporting the results are available at NCBI under GEO accession number GSE133322.

Transcriptome data functional annotation. For functional annotation analysis, BLAST was used to align the assembled unigenes with five public databases. Overall, 23,329 (73.41\%) unigenes could be aligned in one or more databases: 21,079 $(66.33 \%)$ unigenes were similar to proteins in the NR database and 17,398 (54.75\%) were annotated in Swiss-Prot (Table III). For GO annotation, 10,542 unigenes were assigned to the 'cellular component' category and 'integral membrane component' $(n=2,808)$, 'nucleus' $(n=1,604)$ and 'cytosol' ( $\mathrm{n}=1442)$ were other main subcategories. In addition, 11,834 unigenes were classified as 'biological processes'; the three main categories were 'metabolic process' $(n=1,373)$, 'oxidation-reduction process' $(n=1,117)$ and 'transmembrane transport' (706). Furthermore, 12,149 unigenes were assigned to the 'molecular function' category and genes assigned to 'ATP binding' $(\mathrm{n}=1,588)$ and 'metal ion binding' (747) accounted for the vast majority of this category. In the KOG classification, 13,854 unigenes were categorized into 25 KOG functional groups; 'General function prediction only' was the largest group, followed by 'Post-translational modification, protein turnover, chaperones' and 'Translation, ribosomal structure and biogenesis'. In the KEGG pathway analysis, 11,062 unigenes could be mapped to 342 metabolic pathways and the largest category was 'Carbon metabolism' $(\mathrm{n}=424)$.

Analysis of transcriptome expression in the mycelium and 48 - $h$ induced yeast forms. The sequences of sample reads and unigenes were compared using bowtie 2; this aligned 22,315,744 $(89.00 \%)$ reads of the mycelial phase and 19,552,494 (85.00\%) reads of the 48-h induced yeast phase to unigene sequences.

Next, DEGs were identified by comparing the FPKM values for each gene between the mycelial and 48-h induced yeast forms of $S$. schenckii, and DEGs between the two forms were identified. The results demonstrated that 12,217 genes were expressed differentially between the two forms, including 7,271 upregulated and 4,946 downregulated genes (Fig. 3).

GO analysis of DEGs. To identify the major functional categories represented by DEGs, with all unigene genes as background genes, the P-value was calculated using hypergeometric distribution method, and $\mathrm{P}<0.05$ was taken as the threshold to obtain significant heights relative to the background (29). Among DEGs between the mycelial and 48-h induced yeast forms, GOs associated with ATP binding, metal ion binding and zinc ion binding in the molecular function category; GOs associated with metabolic processes, oxidation reduction reactions and transmembrane transport in the biological process category; and particularly those associated with integral components of the membrane, nucleus, cytosol, 


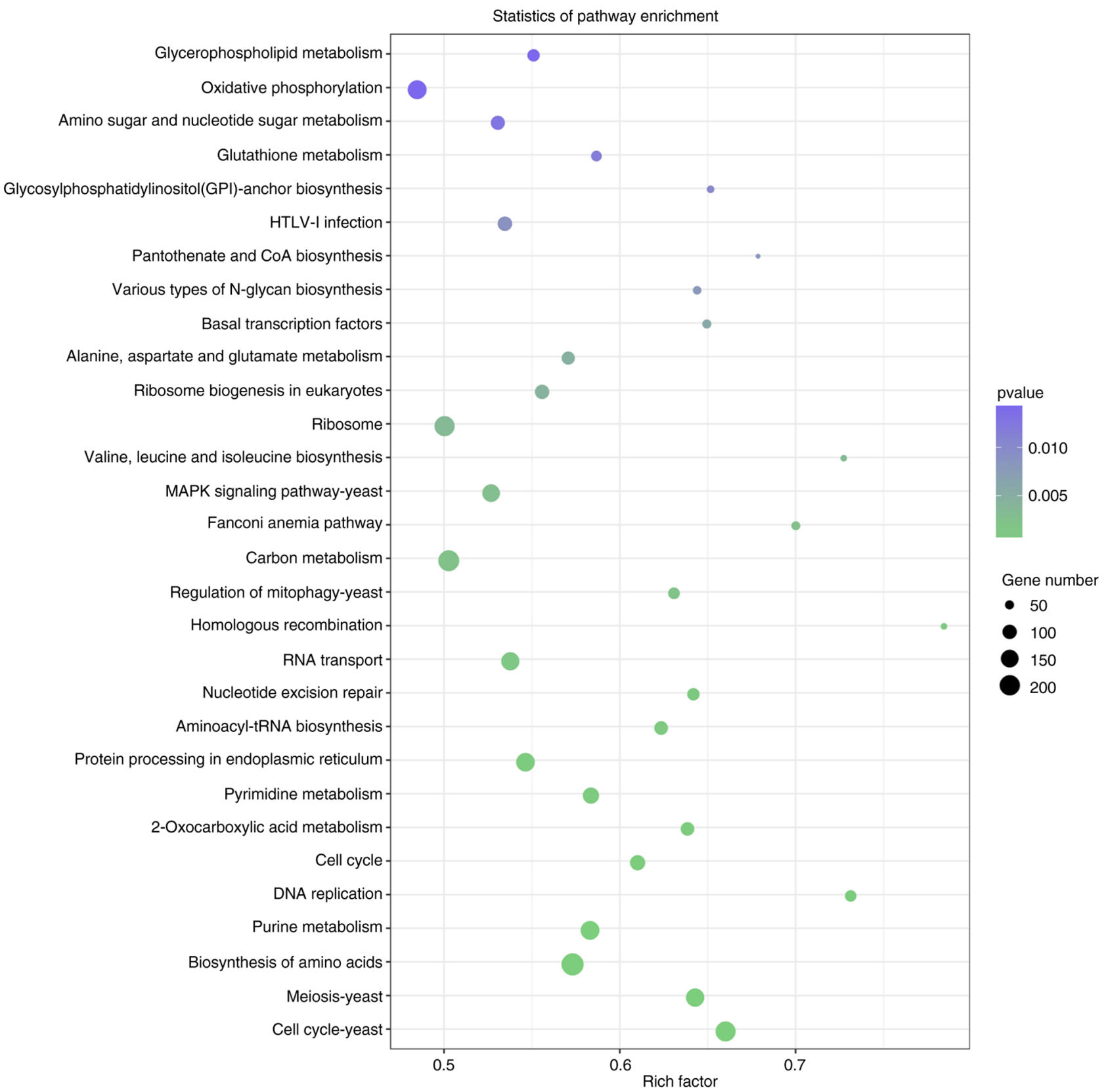

Figure 5. Histogram of the Kyoto Encyclopedia of Genes and Genomes pathway classification of differentially-expressed genes of Sporothrix schenckii.

cytoplasm and mitochondrion in the cellular component group were significantly changed (Fig. 4).

During the process of switching, the form and function of the cell change notably, and those changes must be supported by changes in cell components, including cytoplasmic proteins, nucleoproteins, regulatory proteins, toxic proteins and cell wall component proteins. This led to the observation that genes associated with the integral components of the membrane changed most significantly. These changes require large amounts of protein and energy for anabolism, as well as raw materials absorbed from the environment; therefore, genes associated with metabolic processes, oxidation-reduction processes, transmembrane transport, and the steps of transcription and translation, including ATP binding, metal ion binding, zinc ion binding and DNA binding, changed markedly.
KEGG pathway enrichment of DEGs. To further elucidate the molecular interactions among DEGs, KEGG analysis was performed (30). Among DEGs between the mycelial and 48-h induced yeast forms of S. schenckii, pathways for oxidative phosphorylation, ribosome, the MAPK pathway, carbon metabolism, RNA transport, protein processing in endoplasmic reticulum, pyrimidine metabolism, purine metabolism, biosynthesis of amino acids, meiosis and the cell cycle were enriched (Fig. 5). During the process of switching, enormous changes must occur in the expression of the genes in these pathways, which are necessary for transcription, translation, biosynthesis and energy synthesis. Beyond those predictable changes, it was also found that numerous genes, including DRK1, Hog1, Skn7 and Ste11, which are involved in the two-component system heterotrimeric $\mathrm{G}$ protein, cAMP and Ras-Hog1 signaling pathways, 
Table IV. Genes in signaling pathways associated with dimorphic switching identified in the transcriptome of S. schenckii.

\begin{tabular}{|c|c|c|}
\hline Gene & $\log \mathrm{FC}$ & P-value \\
\hline $\mathrm{CDC} 24$ & 12.49721754 & $3.88 \times 10^{-17}$ \\
\hline $\operatorname{cdc} 28$ & -2.38905262 & $1.72 \times 10^{-3}$ \\
\hline $\operatorname{cdc} 42$ & 12.12659431 & $3.49 \times 10^{-24}$ \\
\hline chk1 & 10.97359207 & $5.54 \times 10^{-24}$ \\
\hline cla4 & 11.87930169 & $2.61 \times 10^{-15}$ \\
\hline drk1 & 8.876696836 & $5.43 \times 10^{-07}$ \\
\hline gpd1 & 10.2951049 & $9.25 \times 10^{-11}$ \\
\hline gre2 & 11.98573676 & $9.21 \times 10^{-24}$ \\
\hline hog1 & 13.35994596 & $1.06 \times 10^{-19}$ \\
\hline hsl1 & 12.78354566 & $5.48 \times 10^{-18}$ \\
\hline $\operatorname{msn} 2,4$ & 13.46928334 & $5.00 \times 10^{-20}$ \\
\hline opy2 & 10.87439311 & $2.16 \times 10^{-12}$ \\
\hline pbs2 & 10.66018548 & $8.56 \times 10^{-20}$ \\
\hline ralbp1 & 2.161074365 & $1.65 \times 10^{-3}$ \\
\hline skn7 & 3.663787304 & $3.75 \times 10^{-06}$ \\
\hline sko1 & 12.57430068 & $2.29 \times 10^{-17}$ \\
\hline $\sin 1$ & 14.1849827 & $3.60 \times 10^{-22}$ \\
\hline ssk1 & -3.84358211 & $3.69 \times 10^{-05}$ \\
\hline ssk2 & 12.08651326 & $1.00 \times 10^{-26}$ \\
\hline ste11 & 3.923507879 & $1.46 \times 10^{-07}$ \\
\hline ste 12 & -3.680198317 & $5.13 \times 10^{-07}$ \\
\hline ste 20 & 13.90389877 & $2.50 \times 10^{-21}$ \\
\hline ste 50 & 11.67340917 & $1.49 \times 10^{-27}$ \\
\hline ste 7 & 12.45175501 & $5.34 \times 10^{-17}$ \\
\hline swe1 & 9.460635913 & $1.83 \times 10^{-08}$ \\
\hline Tcsa & -2.369133725 & $1.06 \times 10^{-2}$ \\
\hline Ypd1 & 10.77251106 & $3.94 \times 10^{-20}$ \\
\hline
\end{tabular}

were altered (Table IV; Fig. 6). These signal transduction pathways serve important roles during the initiation of the dimorphic switch.

\section{Discussion}

To date, little is known regarding the factors driving the pathogenesis of sporotrichosis despite its worldwide prevalence, which hinders medical control of this disease (31). Similar to other pathogenic fungi, host signals that trigger the dimorphic switch are transmitted via signaling pathways and ultimately culminate in changes in gene expression. However, the network of signaling pathways involved in dimorphic switching in S. schenckii remain enigmatic. The present study found that this species exhibits the morphological and structural characteristics of the 48-h induced yeast phase when cultured in BHI medium at $37^{\circ} \mathrm{C}$ for $48 \mathrm{~h}$. Therefore, the transcription profile of that culture was compared with that of the same strain in the mycelial phase using an RNA-seq method. The results of the present study revealed that more than 12,217 genes were significantly upregulated or downregulated in the early yeast phase. Among these genes, many encode signal transduction

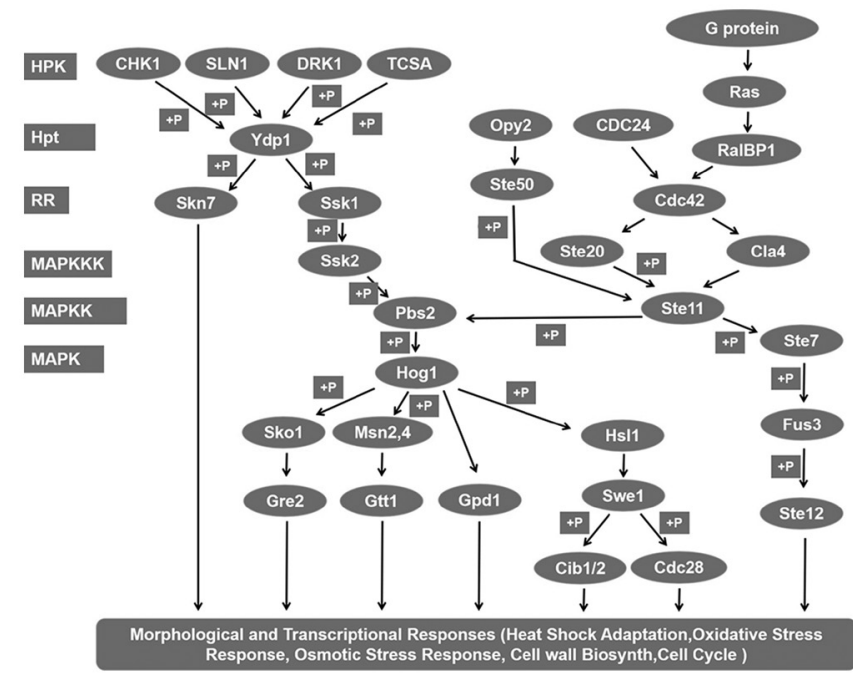

Figure 6. Derived model of the two-component and Ras-Hog1 signaling pathways involved in the initial phase of dimorphic switch of Sporothrix schenckii based on the results of transcriptomic profiling of differentially expressed genes in the 48-h induced yeast phase and the Kyoto Encyclopedia of Genes and Genomes pathway database.

proteins in the two-component and heterotrimeric $\mathrm{G}$ protein, Ras and cAMP signaling pathways and the MAPK cascade, suggesting that these signal transduction pathways serve important roles during the initiation of the dimorphic switch. The results of the present study provided a molecular basis for the development of $S$. schenckii control strategies targeting genes in signaling pathways associated with the dimorphic switch.

In fungi, the two-component system comprises a membrane-associated histidine kinase (HK) and a cytoplasmic response regulator ( $\mathrm{RR})$, also known as a hybrid HK (HHK). The HK perceives an environmental stimulus and is autophosphorylated at a conserved histidine in the kinase domain. Next, the phosphate group is transferred to a conserved aspartate in the receiver domain of the RR, and further phosphorelay occurs through an additional phosphotransfer protein (HPt) and a second response regulator. To date, 11 classes of HHKs (I-XI) in fungi have been identified that are involved in signal phosphorylation and transmission to two RRs, orthologous to Ssk1 and Skn7, via a single HPt, orthologous to Ypd1 (32). The results of the present study revealed that four HKs, orthologous to CHK1 (class VIII), Sln1 (class VI), DRK1 (class III) and TCSA (class IV), as well as two RRs, orthologous to Ssk1 and Skn7, were significantly upregulated or downregulated in the 48-h induced yeast stage compared with the mycelial stage, indicating that they are involved in inducing the dimorphic switch in S. schenckii. Further KEGG analysis suggested that all four HHKs, orthologous to CHK1, SIn1, DRK1 and TCSA, could phosphorylate Ssk1/Skn7 via Ypd1, resulting in constitutive activation of the downstream Hog1 MAPK pathway. Several studies, including our previous study focusing on class III HHK (Os-1 ortholog) in human dimorphic pathogens, have revealed conserved roles in fungicide resistance, osmotic stress resistance and pathogenicity, in addition to roles in cell wall integrity, asexual development and dimorphism $(6,17,33,34)$. Furthermore, SInA (class VI) 
in T. marneffei, Chk1 (class VIII) in C. albicans, and TcsA (class IV) in Aspergillus nidulans were revealed to be associated with osmotic stress resistance, HOG MAPK regulation, the dimorphic transition and development under standard growth conditions (7,35). As multiple histamine kinases may compensate for each other in S. schenckii (Fig. 2), SsDRK1 interference is confirmed to inhibit infection but not kill the pathogenic yeast (17). Therefore, research efforts should focus on pivotal downstream genes, including Pbs2 or Hog1, deletion of which may be lethal, as targets for novel drug development. Our group are creating Pbs2 and Hog1 mutant strains to further investigate the function of vital genes in controlling the dimorphic switch of S. schenckii and will publish the results in a future study.

Heterotrimeric G proteins and the downstream Ras signaling pathways have been demonstrated to influence dimorphic switching and adaptation to oxidative stress in dimorphic fungi, in addition to regulating asexual development and conidial germination $(8,9,13,36)$. Canonical heterotrimeric $G$ proteins comprise three subunits $(\alpha, \beta$ and $\gamma)$ and transmit signals from cell surface receptors. The S. schenckii Ssg-1 Ga subunit interacts with proteins that are necessary for survival under oxidative stress conditions and for iron acquisition, and the Ssg-2 Ga subunit interacts with cytosolic phospholipase A 2, which stimulates the yeast-to-hyphal dimorphic switch and prevents re-entry into the yeast cell cycle $(8,9)$. Several genes in the Ras signaling pathway, including Ras, Rho GTPase Cdc42 and the p21 activated kinases Ste20 and Cla4, were significantly upregulated in the yeast culture, suggesting that signal transmission via these genes from heterotrimeric G proteins occurs during the early stage of the dimorphic switch. In response to stimulation from heterotrimeric G proteins, GTPase-activating proteins convert GTPases of the Ras superfamily from an inactive GDP-bound form to an active GTP-bound form. Further KEGG analysis in the present study indicated that activated Ras activates the Rho GTPase Cdc42, a member of the Ras GTPase superfamily, which regulates the MAPK pathway via Ste20 or Cla4. The results of the present study further support the proposal that $\mathrm{Cdc} 42$ serves a conserved role in regulating morphogenesis by controlling actin-mediated polarized growth and signaling pathways that are required for morphological responses in a variety of fungi (37-39).

Cell wall glycoconjugates of pathogenic fungi, known as pathogen-associated molecular patterns, or PAMPs, are involved in virulence and pathogenicity. Previous studies have reported that the $S$. schenckii cell wall is composed of a peptido-rhamnomannan component, $\beta$-glucans and chitin, which are involved in the innate immune response, binding to the corresponding pattern-recognition receptors and triggering the secretion of specific cytokines (40-42). In the present study, fluorescence microscopy of 48-h cultured yeast cells labeled with calcofluor white to target chitin exhibited a stronger fluorescence labeling pattern than that of mycelial cells, which may indicate a thicker cell wall at the ultrastructural level during the initial yeast phase (43). Chitin, a $\beta(1,4)$-linked homopolymer of $\mathrm{N}$-acetylglucosamine, is an essential component of the cell walls and septa of all pathogenic fungi, and is therefore considered an attractive target for antifungal therapies (44). A variable proportion of fungal chitin is synthesized and then deacetylated to chitosan through the action of one or more chitin deacetylases. Consistent with the fluorescence labeling findings, the transcriptome analysis in the present study indicated that several genes involved in chitin synthesis metabolism were distinctly upregulated in 48 -h induced yeast cells. In addition, activation of the HOG MAPK cascade contributed toward the increased level of chitin in S. schenckii; previous studies have demonstrated that HOG MAPK cascades regulate chitin synthase gene expression and chitin synthesis in response to cell wall stresses in two other dimorphic fungi, S. cerevisiae and C. albicans $(45,46)$.

Taken together, the transcriptome data and analysis in the present study lay the foundation for further research into the molecular mechanisms controlling the dimorphic switch of S. schenckii and support the development of anti-S. schenckii strategies targeting genes associated with signaling pathways.

The present study is limited by the fact that the early yeast Sporothrix cells preparation was performed in the BHI instead of in an animal model. Although the most frequently reported yeast-like Sporothrix cells are grown in BHI, the influence of culture media on the phenotypical trait cannot be ignored.

\section{Acknowledgements}

Not applicable.

\section{Funding}

This study was supported by National Natural Science Foundation of China (grant no. 81472891) and Shenzhen Science and Technology Innovation Commission Project (grant no. JCYJ20180306173356306) which were distributed to Hong Kong University Shenzhen Hospital.

\section{Availability of data and materials}

The transcriptomic data supporting the results of this article are available at NCBI under GEO with accession number GSE133322 (https://www.ncbi.nlm.nih.gov/geo/query/acc. cgi?acc=GSE133322).

\section{Authors' contributions}

$\mathrm{ZZ}$ and FZ designed the study, wrote the article and confirm the authenticity of all the raw data. WG, QC and YW performed the experiments, and QZ, XJ and BH analyzed the data. All the authors read and approved the final version of this manuscript.

\section{Ethics approval and consent to participate}

Not applicable.

\section{Patient consent for publication}

Not applicable. 


\section{Competing interests}

The authors declare that they have no competing interests.

\section{References}

1. Chakrabarti A, Bonifaz A, Gutierrez-Galhardo MC, Mochizuki T and Li S: Global epidemiology of Sporotrichosis. Med Mycol 53: 3-14, 2015.

2. Lopes-Bezerra LM, Mora-Montes HM, Zhang Y, Nino-Vega G, Rodrigues AM, de Camargo ZP and de Hoog S: Sporotrichosis between 1898 and 2017: The evolution of knowledge on a changeable disease and on emerging etiological agents. Med Mycol 56 (Suppl 1): S126-S143, 2018

3. Arenas R, Sánchez-Cardenas CD, Ramirez-Hobak L, Ruíz Arriaga LF and Vega Memije ME: Sporotrichosis: From $\mathrm{KOH}$ to molecular biology. J Fungi (Basel) 4: 62, 2018.

4. Bruno VM, Wang Z, Marjani SL, Euskirchen GM, Martin J, Sherlock G and Snyder M: Comprehensive annotation of the transcriptome of the human fungal pathogen Candida albicans using RNA-seq. Genome Res 20: 1451-1458, 2010.

5. Boyce KJ and Andrianopoulos A: Fungal dimorphism: The switch from hyphae to yeast is a specialized morphogenetic adaptation allowing colonization of a host. FEMS Microbiol Rev 39: 797-811, 2015.

6. Nemecek JC, Wüthrich M and Klein BS: Global control of dimorphism and virulence in fungi. Science 312: 583-588, 2006.

7. Boyce KJ, Schreider L, Kirszenblat L and Andrianopoulos A The two-component histidine kinases $\operatorname{DrkA}$ and $\operatorname{Sin} A$ are required for in vivo growth in the human pathogen Penicillium marneffei. Mol Microbiol 82: 1164-1184, 2011.

8. Valentín-Berríos S, González-Velázquez W, Pérez-Sánchez L, González-Méndez R and Rodríguez-Del Valle N: Cytosolic phospholipase A2: A member of the signalling pathway of a new $\mathrm{G}$ protein alpha subunit in Sporothrix schenckii. BMC Microbiol 9: 100, 2009.

9. Pérez-Sánchez L, González E, Colón-Lorenzo EE, González-Velázquez W, González-Méndez R and Rodríguez-del Valle N: Interaction of the heterotrimeric G protein alpha subunit SSG-1 of Sporothrix schenckii with proteins related to stress response and fungal pathogenicity using a yeast two-hybrid assay. BMC Microbiol 10: 317, 2010.

10. Boyce KJ, Schreider L and Andrianopoulos A: In vivo yeast cell morphogenesis is regulated by a p21-activated kinase in the human pathogen Penicillium marneffei. PLoS Pathog 5: e1000678, 2009.

11. Chen D, Janganan TK, Chen G, Marques ER, Kress MR, Goldman GH,Walmsley AR andBorges-Walmsley MI:The cAMP pathway is important for controlling the morphological switch to the pathogenic yeast form of Paracoccidioides brasiliensis. Mol Microbiol 65: 761-779, 2007.

12. Almeida AJ, Cunha C, Carmona JA, Sampaio-Marques B, Carvalho A, Malavazi I, Steensma HY, Johnson DI, Leão C, Logarinho E, et al: Cdc42p controls yeast-cell shape and virulence of Paracoccidioides brasiliensis. Fungal Genet Biol 46: 919-926, 2009

13. Zuber S, Hynes MJ and Andrianopoulos A: The G-protein alpha-subunit $\mathrm{GasC}$ plays a major role in germination in the dimorphic fungus Penicillium marneffei. Genetics 164: 487-499, 2003.

14. Zhang Z, Hou B, Xin Y and Liu X: Protein profiling of the dimorphic, pathogenic fungus, Sporithrix schenckii. Mycopathologia 173: 1-11, 2012.

15. Hou B, Zhang Z, Zheng F and Liu X: Molecular cloning, characterization and differential expression of DRK1 in Sporothrix schenckii. Int J Mol Med 31: 99-104, 2013.

16. Zhang Z, Hou B, Wu YZ, Wang Y, Liu X and Han S: Two component histidine kinase $D R K 1$ is required for pathogenesis in Sporothrix schenckii. Mol Med Rep 17: 721-728, 2018.

17. Zhang Z, Hou B, Zheng F, Yu X and Liu X: Molecular cloning, characterization and differential expression of a Sporothrix schenckii STE20-like protein kinase SsSte20. Int J Mol Med 31 $1343-1348,2013$

18. Lozoya-Pérez NE, Clavijo-Giraldo DM, Martínez-Duncker I, García-Carnero LC, López-Ramírez LA, Niño-Vega GA and Mora-Montes HM: Influences of the culturing media in the virulence and cell wall of Sporothrix schenckii, sporothrix brasiliensis, and sporothrix globosa. J Fungi (Basel) 6: 323, 2020.
19. de Almeida JRF, Jannuzzi GP, Kaihami GH, Breda LCD, Ferreira KS and de Almeida SR: An immunoproteomic approach revealing peptides from sporothrix brasiliensis that induce a cellular immune response in subcutaneous sporotrichosis. Sci Rep 8: 4192, 2018.

20. Della Terra PP, Rodrigues AM, Fernandes GF, Nishikaku AS, Burger E and de Camargo ZP: Exploring virulence and immunogenicity in the emerging pathogen sporothrix brasiliensis. PLoS Negl Trop Dis 11: e0005903,2017.

21. Kong X, Xiao T, Lin J, Wang Y and Chen HD: Relationships among genotypes, virulence and clinical forms of Sporothrix schenckii infection. Clin Microbiol Infect 12: 1077-1081, 2006.

22. Teixeira PAC, de Castro RA, Nascimento RC, Tronchin G, Perez Torres A, Lazera M, de Almeida SR, Bouchara JP, Loureiro Y Penha CV and Lopes-Bezerra LM: Cell surface expression of adhesins for fibronectin correlates with virulence in Sporothrix schenckii. Microbiology (Reading) 155 (Pt 11): 3730-3738, 2009

23. Brito MM, Conceição-Silva F, Morgado FN, Raibolt PS, Schubach A, Schubach TP, Schäffer GM and Borba CM: Comparison of virulence of different Sporothrix schenckii clinical isolates using experimental murine model. Med Mycol 45: 721-729, 2007.

24. Garrison RG, Boyd KS and Mariat F: Ultrastructural studies of the mycelium-to yeast transformation of Sporothrix schenckii. J Bacteriol 124: 959-968, 1975.

25. Jiang HY, Zhang JL, Yang JW and Ma HL: Transcript profiling and gene identification involved in the ethylene signal transduction pathways of creeping bentgrass (Agrostis stolonifera) during ISR response induced by Butanediol. Molecules 23: 706 , 2018.

26. Wang X, Gao B, Liu X, Dong X, Zhang Z, Fan H, Zhang L, Wang J, Shi S and Tu P: Salinity stress induces the production of 2-(2-phenylethyl)chromones and regulates novel classes of responsive genes involved in signal transduction in Aquilaria sinensis calli. BMC Plant Biol 16: 119, 2016.

27. Qi X, Fang H, Yu X, Xu D, Li L, Liang C, Lu H, Li W, Chen Y and Chen Z: Transcriptome analysis of JA signal transduction, transcription factors, and monoterpene biosynthesis pathway in response to methyl Jasmonate Elicitation in Mentha canadensis L. Int J Mol Sci 19: 2364, 2018.

28. Han C, Li Q, Chen Q, Zhou G, Huang J and Zhang Y: Transcriptome analysis of the spleen provides insight into the immunoregulation of Mastacembelus armatus under Aeromonas veronii infection. Fish Shellfish Immunol 88: 272-283, 2019

29. Du ZY, Zang J, Tang XD and Guo W; Chinese Orthopaedic Association Bone Oncology Group: Experts' agreement on therapy for bone metastases. Orthop Surg 2: 241-253, 2010.

30. Kanehisa M and Goto S: KEGG: Kyoto encyclopedia of genes and genomes. Nucleic Acids Res 28: 27-30, 2000.

31. Conceição-Silva F and Morgado FN: Immunopathogenesis of human sporotrichosis: What we already know. J Fungi (Basel) 4: 89, 2018.

32. Catlett NL, Yoder OC and Turgeon BG: Whole-genome analysis of two-component signal transduction genes in fungal pathogens. Eukaryot Cell 2: 1151-1161, 2003.

33. Boyce KJ, McLauchlan A, Schreider L and Andrianopoulos A: Intracellular growth is dependent on tyrosine catabolism in the dimorphic fungal pathogen Penicillium marneffei. PLOS Pathog 11: e1004790, 2015.

34. Vargas-Perez I, Sanchez O, Kawasaki L, Georgellis D and Aguirre J: Response regulators SrrA and SskA are central components of a phosphorelay system involved in stress signal transduction and asexual sporulation in Aspergillus nidulans. Eukaryot Cell 6: 1570-1583, 2007.

35. Maksimov V, Wäneskog M, Rodriguez A and Bjerling P: Stress sensitivity of a fission yeast strain lacking histidine kinases is rescued by the ectopic expression of Chk1 from Candida albicans. Curr Genet 63: 343-357, 2017.

36. Zuber S, Hynes MJ and Andrianopoulos A: G-protein signaling mediates asexual development at 25 degree $\mathrm{C}$ but has no effect on yeast-like growth at 37 degree $C$ in the dimorphic fungus Penicillium mameffei. Eukaryot Cell 1: 440-447, 2002.

37. Alspaugh JA, Cavallo LM, Perfect JR and Heitman J: RAS1 regulates filamentation, mating and growth at high temperature of Cryptococcus neoformans. Mol Microbiol 36: 352-365, 2000 . 
38. Leberer E, Harcus D, Dignard D, Johnson L, Ushinsky S, Thomas DY and Schröppel K: Ras links cellular morphogenesis to virulence by regulation of the MAP kinase and cAMP signalling pathways in the pathogenic fungus Candida albicans. Mol Microbiol 42: 673-687, 2001.

39. Fortwendel JR, Zhao W, Bhabhra R, Park S, Perlin DS, Askew DS and Rhodes JC: A fungus-specific ras homolog contributes to the hyphal growth and virulence of Aspergillus fumigatus. Eukaryot Cell 4: 1982-1989, 2005.

40. Erwig LP and Gow NA: Interactions of fungal pathogens with phagocytes. Nat Rev Microbiol 14: 163-176, 2016.

41. Previato JO, Gorin PAJ, Haskins RH and Travassos LR: Soluble and insoluble glucans from different cell types of the human pathogen Sporothrix schenckii. Exp Mycol 3: 92-105, 1979.

42. Lloyd KO and Bitoon MA: Isolation and purification of a peptido-rhamnomannan from the yeast form of Sporothrix schenckii. Structural and immunochemical studies. J Immunol 107: 663-671, 1971.

43. Lopes-Bezerra LM, Walker LA, NiñoVega G, Mora-Montes HM, Neves GWP, Villalobos-Duno H, Barreto L, Garcia K, Franco B, Martínez-Álvarez JA, et al: Cell walls of the dimorphic fungal pathogens Sporothrix schenckii and Sporothrix brasiliensis exhibit bilaminate structures and sloughing of extensive and intact layers. PLoS Negl Trop Dis 12: e0006169. 2018.
44. Lenardon MD, Munro CA and Gow NA: Chitin synthesis and fungal pathogenesis. Curr Opin Microbiol 13: 416-423, 2010.

45. Bermejo C, Rodriguez E, Garcia R, Rodriguez-Pena JM, Rodríguez de la Concepción ML, Rivas C, Arias P, Nombela C, Posas F and Arroyo J: The sequential activation of the yeast HOG and SLT2 pathways is required for cell survival to cell wall stress. Mol Biol Cell 19: 1113-1124, 2008.

46. Munro CA, Selvaggini S, de Bruijn I, Walker L, Lenardon MD, Gerssen B, Milne S, Brown AJ and Gow NA: The PKC, HOG and $\mathrm{Ca} 2+$ signalling pathways co-ordinately regulate chitin synthesis in Candida albicans. Mol Microbiol 63: 1399-1413, 2007.

This work is licensed under a Creative Commons Attribution-NonCommercial-NoDerivatives 4.0 International (CC BY-NC-ND 4.0) License. 